\title{
Noble-gas operation of Micro-Hole and Strip Plate electron multipliers at atmospheric-to-high pressures
}

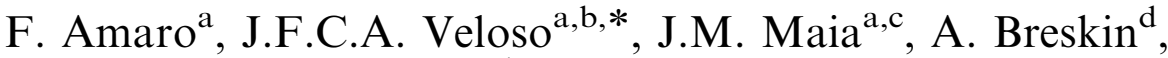 \\ R. Chechik ${ }^{\mathrm{d}}$, J.M.F. dos Santos ${ }^{\mathrm{a}}$ \\ ${ }^{a}$ Physics Department, University of Coimbra, p-3004-516 Coimbra, Portugal \\ ${ }^{\mathrm{b}}$ Physics Department, University of Aveiro, 3810-193 Aveiro, Portugal \\ ${ }^{\mathrm{c}}$ Physics Department, University of Beira Interior, 6200-001 Covilhã, Portugal \\ ${ }^{\mathrm{d}}$ Department of Particle Physics, The Weizmann Institute of Science, 76100 Rehovot, Israel
}

Available online 25 August 2004

\begin{abstract}
We present the performance of a Micro-Hole and Strip Plate (MHSP) electron multiplier in argon-xenon mixtures, at pressures of 1-7 bar. This microstructure can operate at high pressures without significant reduction of the maximum achievable gain. Absolute gains of $1-4 \times 10^{3}$ were reached in $\mathrm{Ar} / 50 \mathrm{mbar}$ Xe over this pressure range; the maximum gain is imposed by the discharge limit, dropping at higher pressures. Energy resolutions between $14 \%$ and $16 \%$ were reached for $6 \mathrm{keV}$ X-rays; they do not degrade significantly with increasing pressure. Better performances are expected by improved manufacturing of the MHSP.
\end{abstract}

(C) 2004 Published by Elsevier B.V.

\section{Introduction}

In recent years, efforts have been made to characterize and improve the performance of micropattern gaseous detectors operated in noble gases and noble-gas mixtures [1-5]. Potential applications are in cryogenic particle detectors for solar neutrino and dark matter research,

\footnotetext{
*Corresponding author. Physics Department, University of Coimbra, p- 3004-516 Coimbra, Portugal. Tel.: + 351-239-410667; fax: + 351-239-829-158

E-mail address: jveloso@gian.fis.uc.pt (J.F.C.A. Veloso).
}

neutron detection, UV-to-visible photon imaging and $\mathrm{X}$-ray detection.

A drawback of the use of micropattern detectors such as MSGCs and GEMs at high pressures is related to the fact that the detector's gain drops with increasing gas pressure [4,6-8]. It is due to the decrease in the reduced electric field, $E / p$, in the avalanche region, limited by the maximum applicable voltage before discharge $[7,8]$. Additionally, secondary photon- and ion-feedback effects often limit the gain. An exception occurs in helium and neon, in which the avalanche is governed predominantly by associative ionisation mechanisms 
rather than by the usual electron impact ionisation [8]. For triple-GEM operation in $\mathrm{Ar}, \mathrm{Kr}$ and $\mathrm{Xe}$, four orders of magnitude gain reduction were observed while increasing pressure from 1 to 5 bar, with gains below 10 at 5 bar [8]. On the other hand, high-pressure operation was reached with a single- GEM in $\mathrm{Kr}$ with the gain decreasing from about 500 to 80 for pressures increasing from 1 to 10 bar [4].

The Micro-Hole and Strip Plate (MHSP) multiplier, shown in Fig. 1 [2,5,9-11], combining in a single structure GEM-like and MSGC-like multipliers, could be a good candidate for noble-gas operation. It has good screening against photon feedback and considerably improved ion-blocking capability as compared to a GEM [11]. It provides good timing and localization properties and can be advantageously placed as the last multiplication element in a multi-GEM cascade. First investigations with an MHSP operating at 1 bar of $\mathrm{Ar}-5 \%$ Xe yielded gains above $10^{4}$ and a $\mathrm{d} E / E$ of $14 \%$, for 5.9-keV X-rays [5].

In this work, we present recent results with MHSP multipliers operating in argon-xenon mixtures, in the pressure range of 1-7 bar. These mixtures permit reaching high gains at relatively

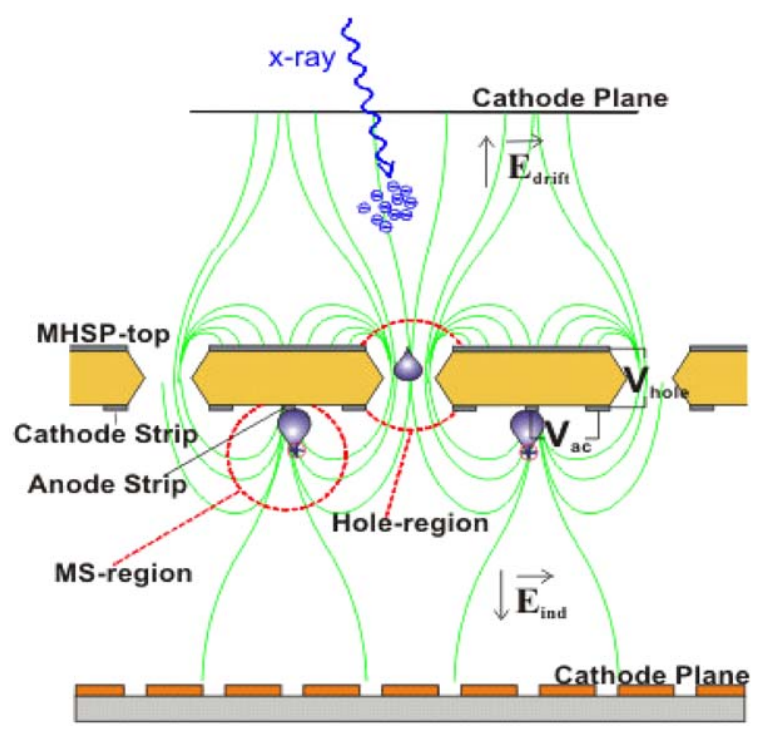

Fig. 1. Schematic diagram of the MHSP and its operating principle. low applied voltages [1,2], which is of particular interest for high-pressure operation.

\section{Experimental set-up}

The MHSP and its operating principle are depicted in Fig. 1. Like the GEM, it is fabricated using printed circuit board technology; the top electrode is unstructured in a GEM-like pattern of bi-conical holes (about $40 / 70 \mu \mathrm{m}$ in diameter), arranged in an asymmetric hexagonal lattice of 140- and 200- $\mu \mathrm{m}$ pitch; and on the bottom surface, a standard MSGC-like pattern is etched, with the holes centred within the cathode strips while the anode strips run between them. The anode and the cathode strips have respective widths of about 35 and $100 \mu \mathrm{m}$. The Kapton substrate foil is $50-\mu \mathrm{m}$ thick.

Electrons induced by radiation in the drift/ absorption region above the MHSP are focused into the holes where they undergo avalanche multiplication, then the avalanche electrons are extracted towards the anode strips where they are further multiplied in a second avalanche process and collected. Most of the ions produced in the avalanche around the anode strips are collected on the neighbouring cathode strips and on the cathode plane localized below the MHSP; only a small fraction of positive ions flows back to the MHSP top electrode and to the drift region, reducing substantially the ion backflow compared to the GEM [11]. The fact that the final avalanche is totally screened by the substrate suppresses totally avalanche-induced photon-feedback. The small distances between the anode and cathode strips result in a fast collection of the avalanche ions, yielding signals with rise-times around 10-30 ns [10].

In the present detector the absorption/drift region and the induction gap are 5 and $3 \mathrm{~mm}$ wide, respectively. Two small non-evaporable getters with built-in heating resistors (SAES St172) were placed inside the detector volume to maintain gas purity. The voltages for the different electrodes of the MHSP are supplied through Macor feedthroughs, which are glued to the voltage wires and to the stainless-steel detector 
body with a low vapour-pressure epoxy (Tra-Con 2116). The detector window is made of an aluminised Mylar foil (25- $\mu \mathrm{m}$ thick) glued to the detector body with the same epoxy. The radiation window, the induction backplane and the detector body are grounded.

Detector signals were fed through a Canberra 2006 preamplifier (with a sensitivity of $1.5 \mathrm{~V} / \mathrm{pC}$ ) and a Tennelec TC243 linear amplifier $(4 \mu \mathrm{s}$ shaping time) to a Nucleus PCA2 1024 multichannel analyser. The electronic chain sensitivity was calibrated using a known charge injected into the preamplifier input.

\section{Experimental results and discussion}

Throughout the measurements the drift field, determined by the MHSP upper electrode voltage, was kept at about $100 \mathrm{~V} / \mathrm{cm}$, and the anodeto-cathode voltage difference, $V_{\text {ac }}$, was kept at $250 \mathrm{~V}$. In each set of measurements, the voltage difference across the holes, $V_{\text {hole, was gradually }}$ increased. To avoid damage to the MHSP, both $V_{\text {hole }}$ and $V_{\text {ac }}$ were maintained below the onset of discharges.

At 1 bar, the best operating conditions, i.e. highest gains for relatively low applied voltages, were obtained with argon $/ \sim 5 \%$ xenon mixtures $[1,2]$. A typical pulse-height distribution for $5.9 \mathrm{keV} \mathrm{X}$-rays is presented in Fig. 2, for a gain of $10^{3}$ at 1 bar of $\mathrm{Ar}-5 \% \mathrm{Xe}$, showing a $\mathrm{d} E / E=13.7 \%$ FWHM and a low background. The energy resolution slightly deteriorates with pressure, reaching values of $\sim 16 \%$ FWHM at 6 bar. In Fig. 3 we present the detector's total gain as a function of $V_{\text {hole, for }} V_{\text {ac }}=250 \mathrm{~V}$, for $\mathrm{Ar}-5 \% \mathrm{Xe}$ mixtures at filling pressures of 1,2 and 3 bar (solid symbols). The curves exhibit the characteristic exponential avalanche growth. As the pressure increases, higher voltages are necessary to achieve similar reduced electric fields and gains; the increase in the energy that the electrons gain from the electric field compensates the increase of energy loss in elastic collisions, as the total pressure rises. The maximum gains, achieved prior to the onset of discharges, present a small decrease from 1 to

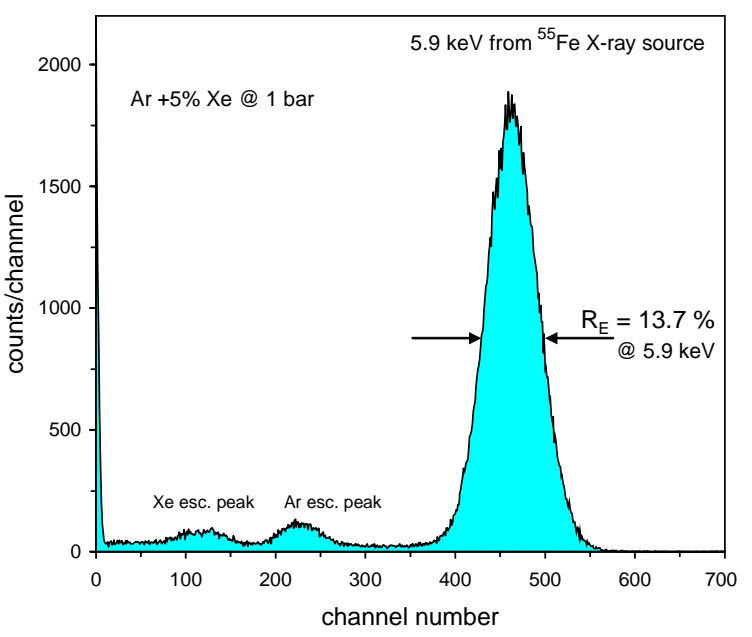

Fig. 2. Typical pulse-height distribution for $5.9 \mathrm{keV}$ X-rays obtained with an MHSP operating in an $\mathrm{Ar}-5 \%$ Xe mixture at 1 bar, at a gain of $10^{3}$.

3 bar, a behaviour similar to that obtained for a GEM operated in $\mathrm{Kr}$ [4].

Since the electron-impact cross-section is higher for Xe than for Ar, the Xe atoms play a more significant role in the charge avalanche processes. Thus, the increase of $\mathrm{Ar}$ content has a smaller effect on the mean-free-path for Xe ionisation, and a constant Xe partial-pressure content could, in principle, yield roughly the same maximum gains. In Fig. 3, the MHSP total gain as function of $V_{\text {hole }}$ is also depicted for $\mathrm{Ar}-\mathrm{Xe}$ mixtures with a constant Xe content ( $50 \mathrm{mbar}$, or $5 \%$ at 1 bar), at filling pressures of 1 (solid symbols), 2 and 3 bar (open symbols). The maximum achievable gain increases slightly, reaching a value of about $3 \times 10^{3}$ at 3 bar $\mathrm{Ar} / 50$ mbar Xe; it is about 5 times higher than that obtained with 3-bar $\mathrm{Ar}-5 \% \mathrm{Xe}$.

In Fig. 4 we depict the MHSP total gain as function of $V_{\text {hole, for }} V_{\mathrm{ac}}=250 \mathrm{~V}$, for $\mathrm{Ar}-\mathrm{Xe}$ mixtures at pressures of $1-7$ bar, with a constant 50 mbar Xe content. The maximum gain is almost constant up to filling pressures of 6 bar, varying between $2 \times 10^{3}$ and $4 \times 10^{3}$. Up to these pressures the increase in the $V_{\text {hole }}$ voltage compensates the increasing energy loss in elastic collisions as the total pressure rises. However, for voltages above $500 \mathrm{~V}$, the $V_{\text {hole }}$ voltage cannot be increased since 


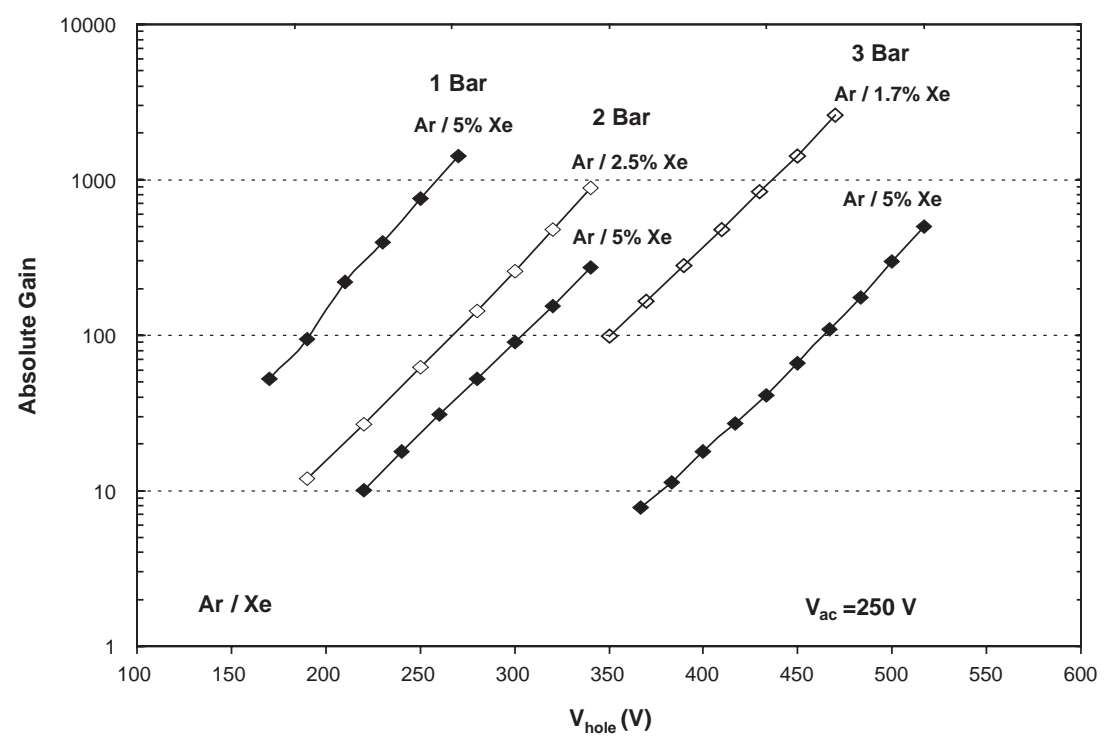

Fig. 3. MHSP total gain as function of the voltage difference across the holes, $V_{\text {hole }}$, for $V_{\text {ac }}=250 \mathrm{~V}$ : (solid symbols) - Ar- $5 \%$ Xe at 1 , 2 and 3 bar; (open symbols) - Ar/50 mbar Xe at 2 and 3 bar.

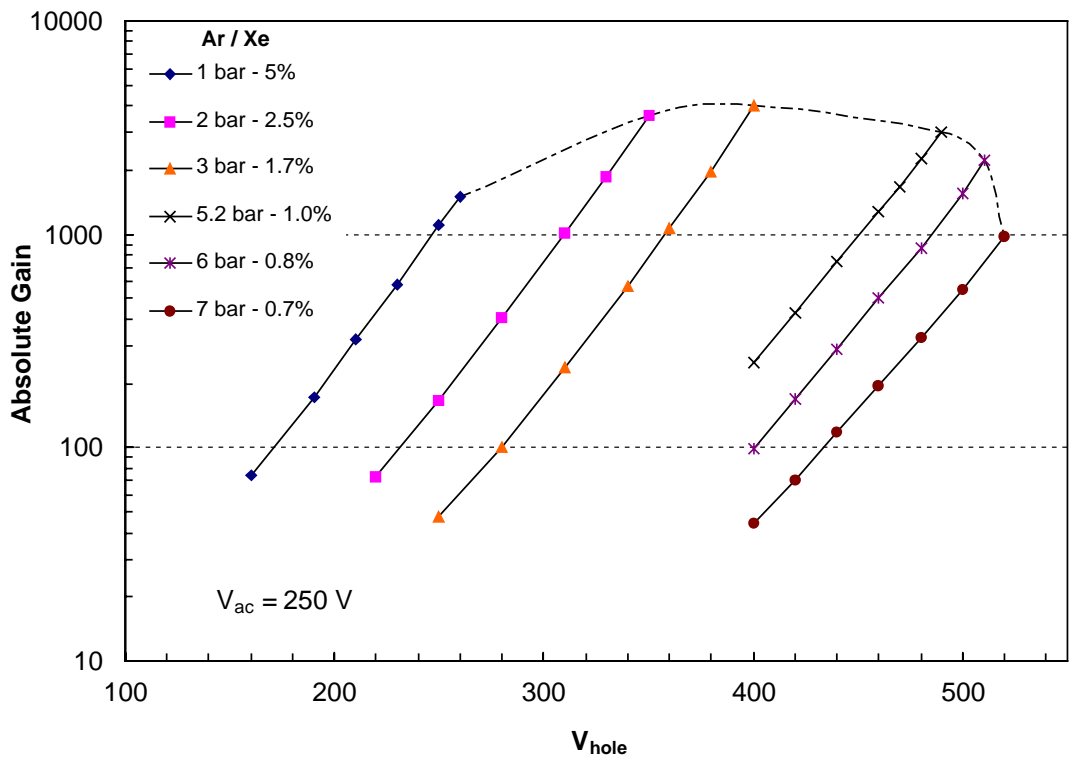

Fig. 4. MHSP total gain as function of the voltage difference across the holes, $V_{\text {hole }}$, for different pressures of Ar/50 mbar Xe.

the electric strength of the microstructure is reaching its discharge limit. In consequence, the compensation can no longer be achieved and the gain drops above this pressure.
In Fig. 5 we present the detector's total gain as function of $V_{\text {hole }}$, for $V_{\mathrm{ac}}=250 \mathrm{~V}$, in pure Xe at 1 and 2 bar. The maximum gain achieved at 1 bar is only a factor of three lower than that obtained with 


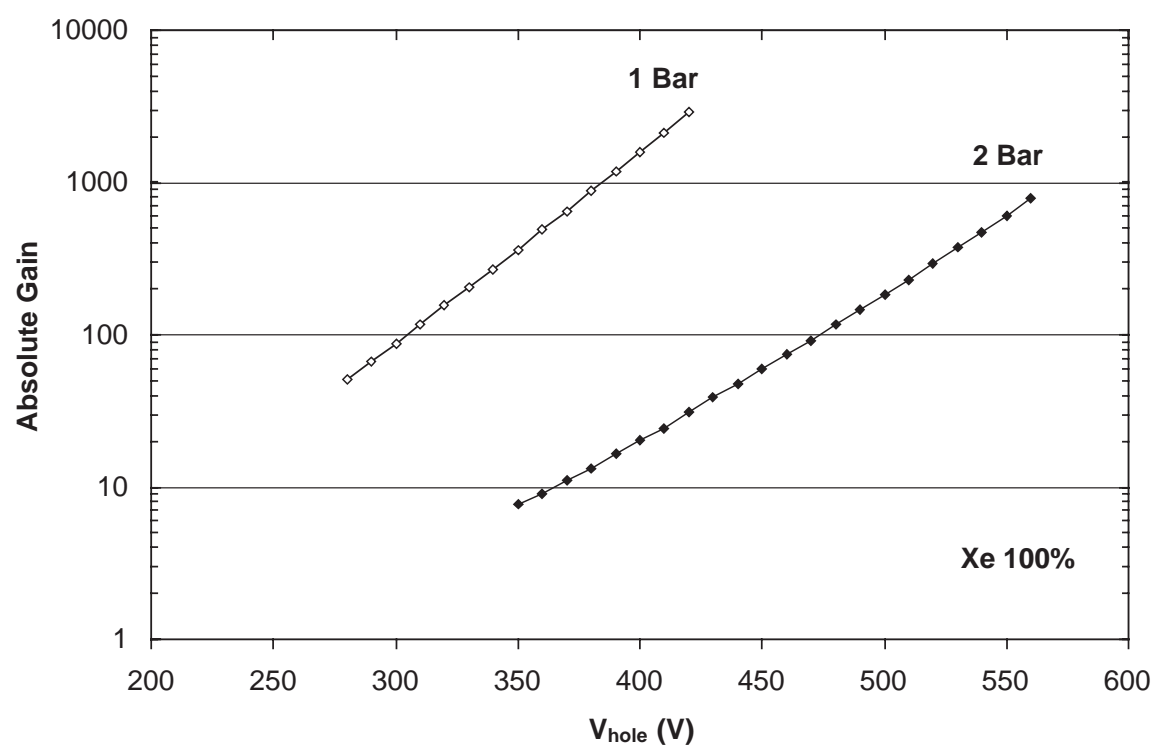

Fig. 5. MHSP total gain as function of the voltage difference across the holes, $V_{\text {hole, }}$ for $V_{\mathrm{ac}}=250 \mathrm{~V}$, in 1 and 2 bar Xe.

a triple-GEM [7] and a factor of six higher than that obtained with a single-GEM in pure Krypton [4].

\section{Conclusions}

We have studied the operation of an MHSP electron multiplier in argon-xenon mixtures, at pressures varying from 1 to 7 bar. High gains, between 2 and $4 \times 10^{3}$ were achieved in $\mathrm{Ar} /$ 50 mbar Xe gas mixtures at pressures up to 6 bar. Above 6 bar, limitations on the maximum gain are imposed by the MHSP discharge limit. Nevertheless, gains are still above $10^{3}$ at 7 bar. Energy resolutions $\sim 14 \%$ FWHM were reached with $6 \mathrm{keV}$ X-rays; they do not degrade significantly with increasing pressure.

It has been demonstrated that, like the GEM [4], a single MHSP could operate at high pressures without significant reduction of the maximum achievable gain. It is expected that improved performance can be achieved when improving manufacturing procedures. Gains of about $3 \times 10^{3}$ were reached with an MHSP operating at $1 \mathrm{bar} \mathrm{Xe}$; it is only a factor of three below that obtained with a triple-GEM. It should be noted that higher gains could be reached in cascading GEMs with an MHSP [11].

\section{Acknowledgments}

Support by FCT, Lisbon (under POCTI and FEDER programmes) under project POCTI/ FNU/49553/02 is gratefully acknowledged. A. Breskin is the W.P. Reuther Professor of Research in the Peaceful Use of Atomic Energy.

\section{References}

[1] A. Buzulutskov, et al., Nucl. Instr. and Meth. A 443 (2000) 164.

[2] J.M. Maia, et al., IEEE Trans. Nucl. Sci. Ns- 49 (2002) 875.

[3] L Periale, et al., Nucl. Instr. and Meth. A 497 (2003) 242 and references therein.

[4] V. Aulchenko, et al., Nucl. Instr. and Meth. A 513 (2003) 256 and references therein.

[5] J.F.C.A. Veloso, et al., Nucl. Instr. and Meth. A 524 (2004) 124. 
[6] A. Bondar, et al., Nucl. Instr. and Meth. A 419 (1998) 418.

[7] A. Bondar, et al., Nucl. Instr. and Meth. A 481 (2002) 200.

[8] A. Bondar, et al., Nucl. Instr. and Meth. A 493 (2002) 8.
[9] J.F.C.A. Veloso, J.M.F. dos Santos, C.A.N. Conde, Rev. Sci. Instrum. 71 (2000) 2371.

[10] J.M. Maia, et al., Nucl. Instr. and Meth. A 504 (2004) 364.

[11] J.M. Maia, et al., Nucl. Instr. and Meth. A 523 (2004) 334. 